\title{
Reply to the letter "Could cardiac troponin I levels predict mortality in the elderly?"
}

\author{
Francesca Attanasio ${ }^{1}$. Pamela Carrer ${ }^{1}$ - . Anna Zurlo ${ }^{1}$ - Anna Rossi ${ }^{1} \cdot$ Luciano Babuin $^{2} \cdot$ Monica Maria Mion $^{3}$.

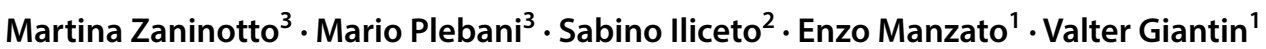

Received: 27 June 2018 / Accepted: 10 July 2018 / Published online: 26 July 2018

(c) Springer Nature Switzerland AG 2018

Dear editor,

We thank Dr. Aycicek and colleagues [1] for their careful reading of our recently published work [2] in which we assessed factors influencing the increase in cardiac Troponin I (cTnI) and its prognostic value in hospitalized older patients.

In our study, we found that cardiovascular diseases (CVD) were statistically more frequent in patients positive on cTnI on admission. This positivity resulted as a mortality risk factor at 6 months after hospital stay. As outlined by the authors, we did not show if CVD were more common in patients who died in comparison with those who survived during the 6-month follow-up. Here we report the results on the prevalence of CVD in these groups of patients, who did not show any significant difference regarding congestive heart failure (23.5 vs. 23.1), coronary heart disease (41.2 vs. 31.1 ), myocardial infarction (23.5 vs. 14.3 ), arrhythmia (33.8 vs. 33.6 deceased vs survived patients in all comparisons).

Concerning having considered CVD in the regression analyses, we confirm that such comorbidities were taken into account by including in our models the variable "Heart diseases", defined as the presence of one among the following: congestive heart failure, coronary heart disease, myocardial infarction, arrhythmias or left ventricular hypertrophy.

With regard to the time elapsed between the initiation of the symptoms and admission to Emergency Department, this data was not collected and we recognize this as a limit of our study.

Pamela Carrer

pamelacarrer@gmail.com

1 Geriatrics Division, Department of Medicine (DIMED), University of Padova, Via Giustiniani, 2, 35128 Padova, Italy

2 Department of Cardiac, Thoracic and Vascular Sciences, University of Padova, Padova, Italy

3 Department of Laboratory Medicine, University-Hospital of Padova, Padova, Italy
In conclusion, we thank Dr. Aycicek and colleagues for their accurate comments. We agree with them that further research is needed in the light of these suggestions to assess the real prognostic value of $\mathrm{cTnI}$ in older patients.

Funding This study did not receive external funding.

\section{Compliance with ethical standards}

Conflict of interest The authors declare that they have no conflict of interest.

Ethical approval All procedures performed in this study were in accordance with the ethical standards of the institutional and national research committee and with the 1964 Helsinki declaration and its later amendments or comparable ethical standard.

Informed consent Informed consent was obtained from all individual participants included in the study.

\section{Reference}

1. Aycicek GS, Unsal P, Esme M, Balci C, Yavuz BB, Halil MG, Cankurtaran M (2018) Could cardiac troponin I levels predict mortality in the elderly? Aging Clin Exp Res. https://doi. org/10.1007/s40520-018-1000-3

2. Attanasio F, Carrer P, Zurlo A, et al (2018) Prognostic value of cardiac troponin I assay in hospitalized elderly patients. Aging Clin Exp Res. https://doi.org/10.1007/s40520-018-0965-2 